\title{
A Seroepidemiological Survey for Pertussis among Adolescents
}

\author{
Lydia Jennifer $\mathrm{S}^{1}$ • Mona M. Basker ${ }^{2}$ - Valsan Philip Verghese ${ }^{2} \cdot$ Shalini Anandan ${ }^{1}$. \\ Dhiviya Prabaa Muthuirulandi Sethuvel ${ }^{1} \cdot$ Sankar Baby Abirami ${ }^{1} \cdot$ Thenmozhi Mani $^{3} \cdot$ Veeraraghavan Balaji $^{1}$. \\ John Antony Jude Prakash ${ }^{1}$
}

Received: 15 September 2020 / Accepted: 5 February 2021 / Published online: 17 January 2021

(C) Dr. K C Chaudhuri Foundation 2021

To the Editor: Pertussis is a highly contagious vaccine preventable disease caused by Bordetella pertussis. Waning of childhood vaccination-induced immunity has resulted in pertussis infection among adolescents in many countries. They, in turn, are a source of infection especially for young children in the community [1]. There is little epidemiological data on the prevalence of pertussis among adolescents in India. Hence, a prospective cross-sectional study was conducted from August 2015 to October 2017. Venous blood samples were collected from adolescents aged 10 to $19 \mathrm{y}$. Immunoglobulin $\mathrm{G}(\mathrm{IgG})$ antibody titer against pertussis toxin (PT) was estimated by enzyme-linked immunosorbent assay (ELISA). Titers $\geq 62.5$ to $<125 \mathrm{IU} / \mathrm{mL}$ and $\geq 125 \mathrm{IU} / \mathrm{mL}$ were considered to correspond to $1 \mathrm{y}$ and 6 mo post pertussis infection, respectively and titer $<5 \mathrm{IU} / \mathrm{mL}$ indicated undetectable levels [2].

A total of 281 subjects were recruited. All had received the primary vaccines and one booster dose with diphtheria, pertussis, tetanus (DPT). Second booster was taken by 120 $(42.7 \%)$ and a third in adolescent age by $15(5.3 \%)$ participants. Anti-PT mean antibody levels were $9.5 \mathrm{IU} / \mathrm{mL}$ (95\% CI 8.4-10.6 IU/mL). Evidence of pertussis infection in the previous year was observed in $11(3.9 \%)$, and in the previous 6 mo in $8(2.8 \%)$, whereas undetectable $\mathrm{IgG}$ were recorded in $153(54.4 \%)$ adolescents.

John Antony Jude Prakash

prakjaj@cmcvellore.ac.in

1 Department of Clinical Microbiology, Christian Medical College, Vellore, Tamil Nadu 632004, India

2 Department of Pediatrics, Christian Medical College, Vellore, Tamil Nadu, India

3 Department of Biostatistics, Christian Medical College, Vellore, Tamil Nadu, India
In the present study, around $7 \%$ of adolescents had evidence of recent infection and they could act as a reservoir to unimmunized or partially immunized infants and children [3]. This is significant as $54 \%$ of the adolescents tested had no detectable antibodies, suggesting waning immunity and susceptibility to pertussis, which can lead to periodic epidemics as suggested by Campbell et al. [4]. These findings suggest that, administration of one booster dose of pertussis vaccine to all Indian adolescents, as recommended by vaccine advisory bodies is justified [1].

\section{Declarations}

Ethical Clearance The study was approved by the Institutional Review Board, Christian Medical College, Vellore (IRB Min No: 8348 dated 26.06.2013).

Conflict of Interest None.

Funding Institutional Review Board, Christian Medical College, Vellore (IRB Min No: 8348, 26.06.2013).

\section{References}

1. Pinkbook. Pertussis. Epidemiology of Vaccine Preventable Diseases. CDC. 2019. Available at: https://www.cdc.gov/vaccines/pubs/ pinkbook/pert.html. Accessed 06 April 2020.

2. Jõgi P, Oona M, Toompere K, Leedo S, Epstein J, Lutsar I. Seroprevalence of $\mathrm{IgG}$ antibodies to pertussis toxin in children and adolescents in Estonia. Vaccine. 2014;32(41):5311-5.

3. Skoff TH, Kenyon C, Cocoros N, et al. Sources of infant pertussis infection in the United States. Pediatrics. 2015;136(4):635-41.

4. Campbell P, McIntyre P, Quinn H, Hueston L, Gilbert GL, McVernon J. Increased population prevalence of low pertussis toxin antibody levels in young children preceding a record pertussis epidemic in Australia. PLoS One. 2012;7(4):e35874.

Publisher's Note Springer Nature remains neutral with regard to jurisdictional claims in published maps and institutional affiliations. 